\title{
An investigation on the phase angle of radio signals from cosmic ray air showers
}

\author{
Mohammad Sabouhi* \\ Department of Physics, Semnan University \\ E-mail: m.sabouhiesemnan.ac.ir
}

\section{Gohar Rastegarzadeh}

Department of Physics, Semnan University

E-mail: grastegarasemnan.ac.ir

\begin{abstract}
It is possible to determine key parameters of a cosmic ray air shower from different approaches. One of the most important elements of an air shower is the place where the shower axis hits the ground, the shower core. Determining the position of this component can result in calculating other important characteristics of a cosmic ray. In this study and based on CORSIKA and COREAS simulations we present a new approach to show the possibility of finding the position of this point by analyzing the slope of the phase angle from radio signals. The simulations were performed for different type of air showers with 0.1 to $1 \mathrm{EeV}$ energy with Proton and Iron as primary particles. In order to consider the effect of the geomagnetic field as the most important mechanism in creation and propagation of radio signals from cosmic ray air showers, we choose two different locations in the South and North hemispheres namely Pierre Auger Observatory and the location of the expected Semnan Radio Array. The result shows a direct relation between the slope of the phase angle and the distance from shower core. We investigate the relation of this parameter to the initial energy, the shower arrival direction and the geomagnetic field value. In all conditions, we calculated the radio signals at the shower core to have on average, the minimum slope as a function of frequency using a specific computer code developed for this study.
\end{abstract}

35th International Cosmic Ray Conference - ICRC2017

10-20 July, 2017

Bexco, Busan, Korea

${ }^{*}$ Speaker. 


\section{Introduction}

There are many different approaches to detect cosmic rays and to determine their key properties either by direct measurements or by investigating the air showers created as a result of a cosmic ray primary particle interacting with the earth atmosphere [1].

Over the past decade, the radio detection of cosmic ray air showers with its advantages including the nearly $100 \%$ duty cycle, an inexpensive setup and the complementary features has been used in many different experiments to determine key parameters of cosmic rays either in a self-trigger setup or as a commentary element with other detection techniques such as particle detectors or fluorescence light detectors [2],[3].

Because of the progress made in this field, it is now possible to determine some of the most important characteristics of a cosmic ray like energy, direction and type of primary particle from radio measurements only [4], [5]. One of such parameters is the place where the shower axis hits the ground, the shower core. Determining the position of this point may lead to specify other important properties of a cosmic ray.

In this study and by analyzing the phase angle parameter of radio signals that we record on the ground, we try to introduce a new possible approach to determine the position of this point.

\section{Simulations}

For this study, a series of computer simulations were performed by CORSIKA 7.5 [6] and CoREAS 1.1 [7]. The simulated cosmic rays produce vertical and inclined air showers with different initial energy from $10^{17}$ to $10^{18} \mathrm{eV}$ with proton and Iron as primary particles. In order to investigate the effect of the earth magnetic field, as the most important mechanism in creation and propagation of radio signals from cosmic ray air showers [8], we perform the simulations in two different locations. First with the earth magnetic field set to correspond with the Pierre Auger Observatory (PAO) [2] and second in a location that we expect to have the Semnan Radio Array (SRA) in near future. The chosen sites are in the South and North hemisphere respectively.

We used a specific computer code, developed for radio studies at the Semnan University, to analyze the simulated radio signals. This code is capable of doing various types of studies on the simulated radio signals. Here we use this code to calculate the normalized phase angle as a function of frequency.

QGSJETII-04 [9] and Gheisha 2002d has been selected as hadronic interaction models for high and low energies in our simulations with Thinning set to $10^{-6}$.

\section{Vertical Cosmic Rays}

In the first step, we investigate vertical cosmic rays with different energies propagating in the location of PAO and SRA. Fig.1 shows the phase angle parameter for a vertical cosmic ray with $10^{17} \mathrm{eV}$ initial energy propagating at the location of SRA. This figures clearly shows that the slope of the phase angle is dependent on the absolute distance from shower core. As we move from shower core to further distances, the slope of the phase angle increase. 
In this example, we calculate the slope of the phase angle in the North direction from shower core. As it is obvious from the right image in Fig.1, the potential accuracy of this method is less than 5 meters for a vertical cosmic ray.
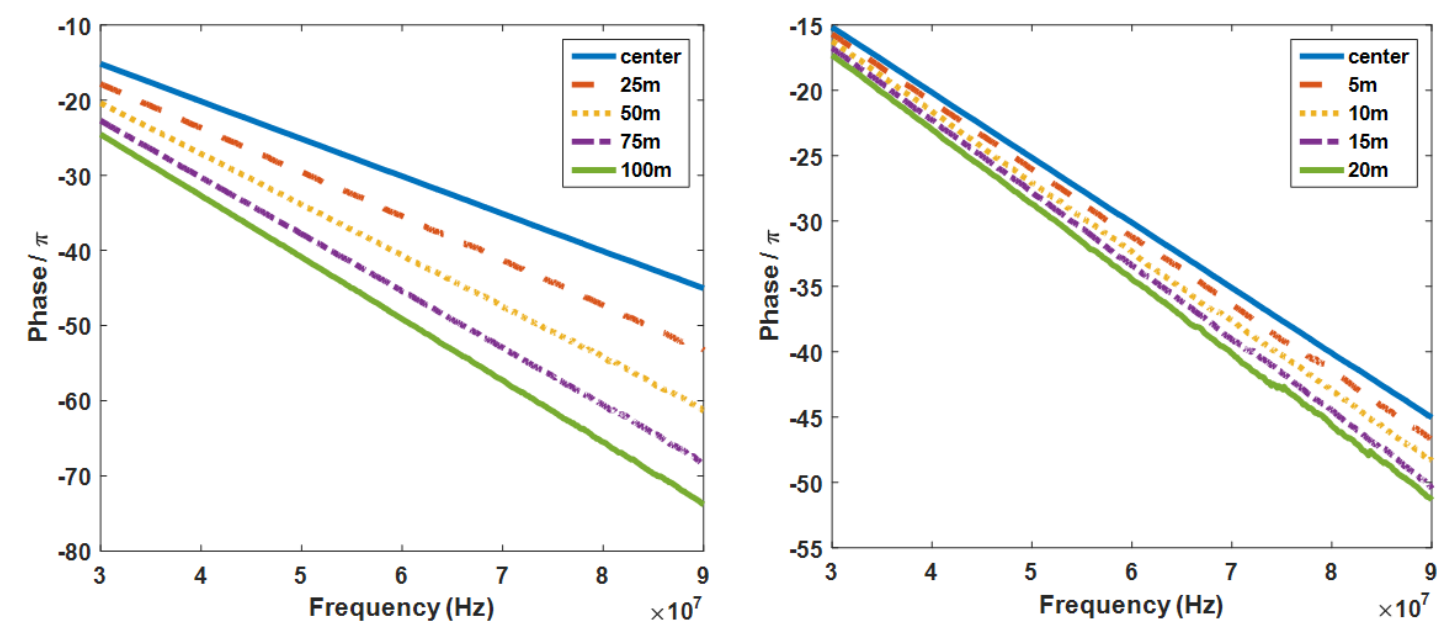

Figure 1: The phase angle for a vertical cosmic ray at the location of SRA. The potential accuracy of this method is less than 5 meters for a vertical cosmic ray.

As it is believed right now, the earth magnetic field plays the major role in the radio signals that are generated from an air shower. This is the reason that we also investigate a vertical cosmic ray and recalculate the slope of the phase angle for a proton cosmic ray with $10^{17} \mathrm{eV}$ energy propagating in the location of PAO. As it can be seen in Fig.2 there is not much difference between the behavior of the phase angle under new circumstances. The slope of the phase angle at the shower center still has the minimum value. Furthermore, Fig.2 also shows that the behavior of this parameter for a vertical cosmic ray only depends on the absolute radial distance from shower core. One important point is that the slope of the phase angle is almost identical for radio signals recorded at different $\theta$ angles from shower core. This can be very helpful to estimate the position of a shower core on a real experiment.

We also investigate a possible effect of the initial energy and type of primary particle on the radio signal phase angles from a vertical cosmic ray. Fig. 3 Shows the slope of the phase angle for a vertical Iron cosmic ray with $10^{18}$ and $10^{17} \mathrm{eV}$ energy from left to right, respectively. In both cases, the slope of the phase angle has on average, the minimum value at shower center. Comparing Fig. 1 to Fig. 3 Shows that the slope of the phase angle is independent not only to primary particle and initial energy but also to the earth magnetic field value for vertical cosmic rays.

It should be noted that all phase angle slopes in this study are based on the East-West polarization component of cosmic ray radio signals.

\section{Inclined Cosmic Rays}

To demonstrate the effect of a cosmic ray propagation direction on the slope of the phase angle, we investigate two inclined proton cosmic ray with $\theta=60^{\circ} . \phi=0^{0}$ and $180^{\circ}$ respectively at 

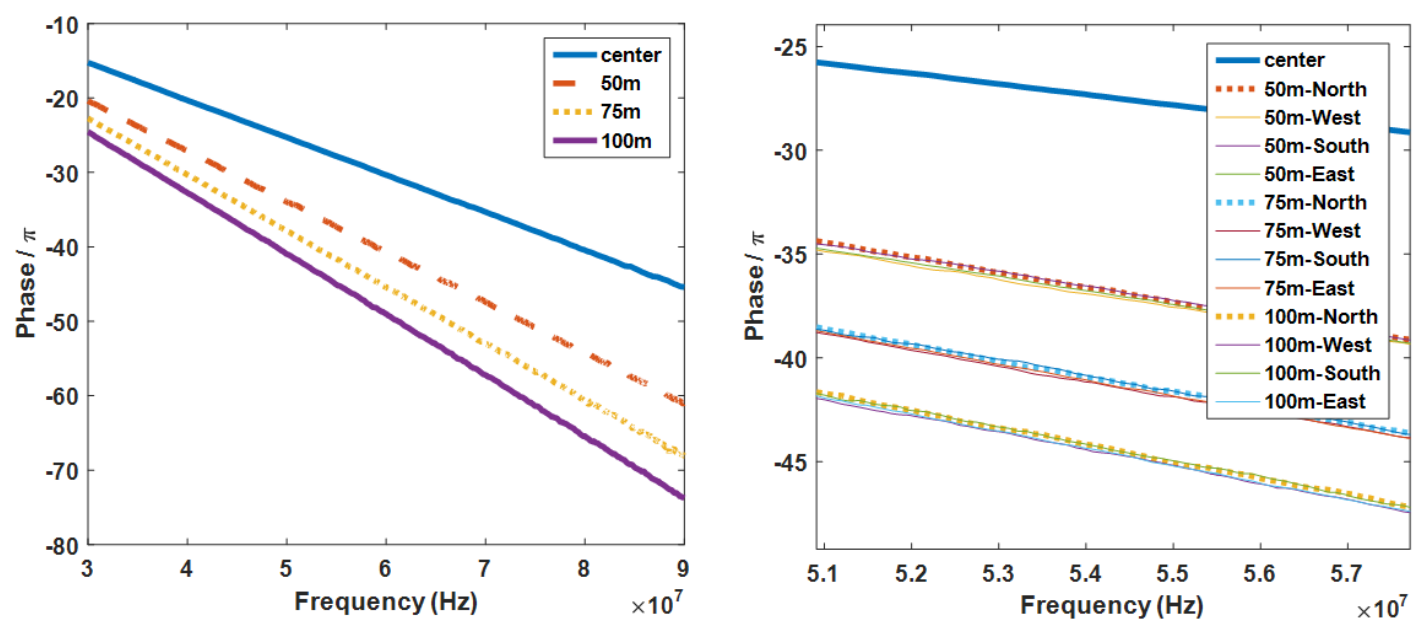

Figure 2: The phase angle for a vertical cosmic ray at the location of PAO. Comparing Fig.1 and Fig.2 shows that the slope of the phase angle has not changed much for a vertical cosmic ray. The dependence of the slope to the absolute distance from shower core is also depicted in the right image.
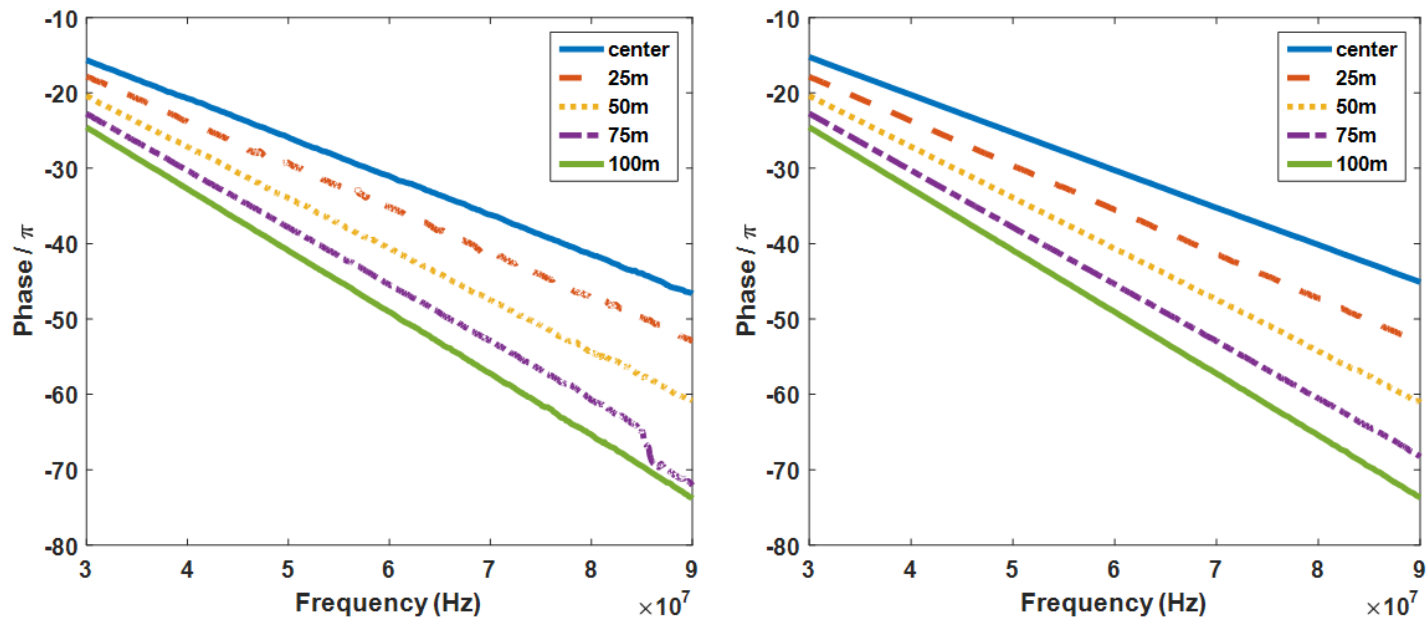

Figure 3: From left to right: the phase angles for an Iron vertical cosmic ray with $10^{17}$ and $10^{18} \mathrm{eV}$ energy at the location of PAO.

the location of SRA and PAO with $10^{17} \mathrm{eV}$ initial energy.

As can be seen in Fig.4, the overall shape of the slope of the phase angle as a function of frequency changes compared to what we calculated in the previous section. The direct line of the phase angle for a vertical cosmic ray now forms differently under new conditions. Moreover, the slopes of the phase angles for inclined cosmic rays are very narrow at different distances from shower core. In this case, even the slope of the phase angle at $50 \mathrm{~m}$ interval is very close to the one belongs to the shower core.

Despite all, the average slope of our intended parameter is calculated to be minimum at the shower center. One important note is the effect of a cosmic ray zenith angle on the slope of the 
phase angle. As the shower becomes more inclined the lines of the slope parameter get closer to each other and that makes it harder to distinguish each specific line. However, even in this case, the higher line belongs to radio signals at a shower core.

The results show that we should expect the same result for cosmic rays with different $\phi$ parameters. Fig. 4 also shows that we should expect to observe similar behavior for cosmic rays propagating to the North or South.

It should be noted that we investigated a situation where the shower core in inside our radio array. A universal approach to determine the position of the shower core is going to be discussed in a later study.
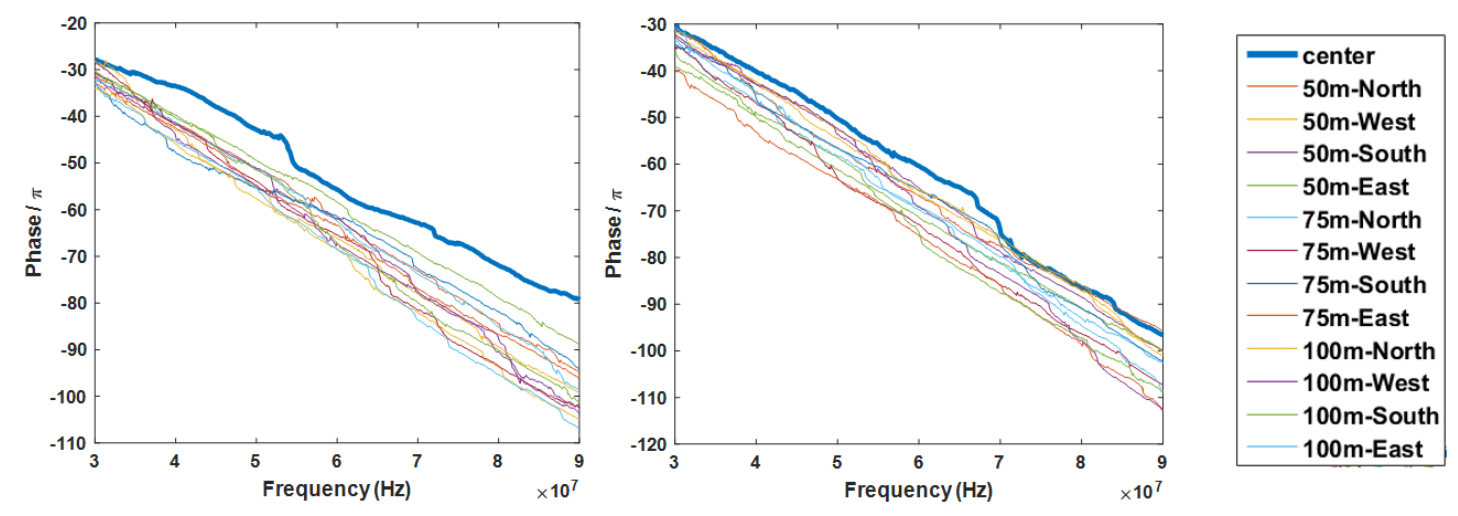

Figure 4: From left to right: the phase angles for an inclined cosmic ray with $10^{17} \mathrm{eV}$ initial energy with $\theta=60^{\circ}, \phi=0^{0}$ and $180^{\circ}$ respectively at the location of SRA and PAO.

\section{Conclusion}

In this study and based on computer simulations, we showed a new possible way to locate the position of a cosmic ray shower center by analyzing the slope of the phase angle from radio signals.

For this purpose, a series of simulations were performed. The simulated cosmic rays had $10^{17}$ to $10^{18} \mathrm{eV}$ initial energy with Proton and Iron as the primary particle propagating in different directions. In order to investigate the effect of the earth magnetic field, we chose two different locations in South and North hemispheres with different magnetic field values, the location of the Pierre Auger Observatory and a location that we expect to have Semnan Radio Array in near future.

The result showed that for vertical cosmic rays there is a significant difference between the calculated slope of the phase angle of radio signals in different distances from shower core. In this case, the lines of phase angles are distinguishable even in $5 \mathrm{~m}$ intervals from shower core. For air showers from vertical cosmic rays, we showed that the slope of the phase angle is independent of the initial energy and type of the primary particle. In all circumstances, the average minimum slope belongs to radio signals at the shower center.

As the shower becomes inclined, the lines of the phase angles at different distances get closer to each other. However, even in this situation, the slope of the phase angle at the shower core has the average minimum slope compared to other lines. 


\section{References}

[1] Karl-Heinz Kampert, Alan A. Watson, Extensive air showers and ultra high-energy cosmic rays: $a$ historical review, The European Physical Journal H 37 (2012) 359.

[2] Pierre Auger Collaboration. (A. Aab et al.), The Pierre Auger Cosmic Ray Observatory, Nucl.Instr.andMeth.A A 798 (2015) 172.

[3] Telescope Array Collaboration. (H. Kawai et al.), Telescope Array Experiment, Nucl.Instr.andMeth.B,Proc. Suppl. 175 (2008) 221.

[4] D. Ardouin, A. BellÂt' etoile, D. Charrier et al., Radioelectric field features of extensive air showers observed with CODALEMA, Astropart. Physics 26 (2006) 341.

[5] T. Huege, R. Ulrich, R. Engel., Theory and simulations of air shower radio emission, Astropart. Physics 30 (2008) 96.

[6] D. Heck, J. Knapp, J.N. Capdevielle, G. Schatz, T. Thouw., CORSIKA: a Monte Carlo code to simulate extensive air showers, FZKA Report 90 (1998) 6019.

[7] T. Huege, M. Ludwig, and C.W. James., Simulating radio emission from air showers with CoREAS, AIP Conf 128 (2013) 1535.

[8] T. Huege, M. Ludwig, O. Scholten, and K.D. de Vries., The convergence of EAS radio emission models and a detailed comparison of REAS3 and MGMR simulations, Nucl. Instr. and Meth. A 662 (2012) S179.

[9] K. D. de Vries, O. Scholten, and K.Werner., Astropart. Phys 45 (2013) 23. 